\title{
Rotational surfaces with Cheng-Yau operator in Galilean 3-spaces
}

\author{
Alev Kelleci (D) \\ Department of Mathematics, Firat University, 23200 Elazı̆̆, Turkey
}

\begin{abstract}
In this paper, we study three types of rotational surfaces in Galilean 3-spaces. We classify rotational surfaces satisfying

$$
L_{1} G=F(G+C)
$$

for some constant vector $C \in \mathbb{G}^{3}$ and smooth function $F$, where $L_{1}$ denotes the Cheng-Yau operator.
\end{abstract}

Mathematics Subject Classification (2020). 53A10, 53A35, 53A40

Keywords. $L_{1}$-pointwise 1-type, Cheng-Yau operator, rotational surface, Galilean spaces

\section{Introduction}

Let $M$ be a hypersurface of the $(n+1)$-dimensional Euclidean space $\mathbb{E}^{n+1}$. A smooth mapping $\phi: M \rightarrow \mathbb{E}^{n}$ is said to be of $k$-type if it can be expressed as a sum of eigenvectors of Laplace operator $\Delta$ corresponding to $k$ distinct eigenvalues of $\Delta[6]$. If $\phi$ is an immersion from $M$ to $\mathbb{E}^{n+1}$ is of $k$-type, then the submanifold $M$ is said to be of $k$-type [3,7]. A good survey on finite type submanifolds is given by Chen in [4].

As a special case of the above definition, one can obtain that $\phi=G$ is of 1 -type if and only if $\Delta G=\lambda(G+C)$ for a constant $\lambda \in \mathbb{R}$, a constant vector $C$ and $G$ is the Gauss map of $M$. On the other hand, if the Gauss map $G$ of $M$ satisfies

$$
\Delta G=F(G+C)
$$

for a non-zero function $F \in C^{\infty}(M)$ and a constant vector $C=\left(C_{1}, C_{2}, \ldots, C_{n}\right)$, then $M$ is said to have pointwise 1-type Gauss map. Many researchers investigated on hypersurfaces with such Gauss map in different ambient spaces $[5,11,13,17]$. Moreover, since the Laplacian operator of a hypersurface $M$ immersed in $\mathbb{E}^{n+1}$ is a second-order linear diferential operator arising naturally as the linearized operator of the first variation of the mean curvature for normal variations of hypersurfaces, so the Laplace operator $\Delta$ can be considered as the first one of a sequence of $n$ operators $L_{0}, L_{1}, L_{2}, \ldots, L_{n-1}$ where $L_{k}$ stands for the linearized operator of the first variation of the $(k+1)$-th mean curvature which arises from normal variations of hypersurfaces for a fixed $k=2,3, \ldots, n-1$, (see more, $[1,12])$. Note that in case $k=0$, the operator $L_{0}=-\Delta$ nothing but the Laplacian operator; when $k=1$ the operator $L_{1}$ is the operator $\square$ introduced by Cheng and Yau in

Email address: alevkelleci@hotmail.com

Received: 29.08.2019; Accepted: 27.06.2020 
[8] and called the Cheng-Yau operator. Recently in [14-16], authors have investigated the surfaces with $L_{1}$-pointwise 1 -type Gauss map in Euclidean 3 -space, $\mathbb{E}^{3}$, defined as

$$
L_{1} G=F(G+C)
$$

for some constant vector $C \in \mathbb{E}^{3}$ and smooth function $F$, where $L_{1}$ denotes the Cheng-Yau operator.

Motivated by the concept of the Cheng-Yau operator of hypersurfaces of Euclidean 3 -space, we would like to give next definition:

Definition 1.1. An admissible surface $M$ in the Galilean space $\mathbb{G}^{3}$ is said to have $L_{1}$-pointwise 1-type Gauss map if its Gauss map satisfies

$$
L_{1} G=F(G+C)
$$

for a smooth function $F \in C^{\infty}(M)$ and a constant vector $C \in \mathbb{G}^{3}$. More precisely, an $L_{1}$-pointwise 1 -type Gauss map is said to be of the first kind if (1.2) is satisfied $C=0$; otherwise, it is said to be of the second kind. Moreover, if (1.2) is satisfied for $F=$ const. (resp., $L_{1} G=0$ ), then we say that $M$ has $L_{1}$-(global) 1 -type Gauss map, (resp., $L_{1}$-harmonic Gauss map).

On the other hand, we are going to use the following lemma obtained for surfaces in $\mathbb{E}^{3}$ in [15]:

Lemma 1.2. Let $M$ be an oriented surface in $\mathbb{E}^{3}$ with the Gaussian curvature $K$ and the mean curvature $H$. Then the Gauss map $G$ of $M$ satisfies

$$
L_{1} G=-\nabla K-2 H K G .
$$

Indeed, the problem of classification of surfaces in Euclidean spaces according to the properties of their curvatures has a long history. Besides Euclidean geometry, a range of new types of geometries, like Minkowski geometry, isotropic geometry, have been invented and developed in the last two centuries. Among these geometries there are also Galilean and pseudo-Galilean geometries. Several articles also appeared on the geometry of some special surfaces in these spaces studied in [2,9,10,18-23].

Note that Yoon, Kim and Jung studied rotation surfaces with $\mathrm{L}_{1}$-pointwise 1-type Gauss map in pseudo-Galilean space $\mathbb{G}_{3}^{1}$, in [24]. They classified only two types of rotational surfaces satisfiying the condition (1.2) in pseudo-Galilean space. But, in [10], the authors showed that there are three types of rotational surfaces in Galilean spaces.

The main interest of this paper is to obtain complete classification of rotational surfaces satisfying (1.2) in the Galilean 3-space $\mathbb{G}^{3}$. In Section 2, we introduce the notations that we are going to use and give a brief summary of basic definitions in theory of surfaces in Galilean spaces. In Section 3, we obtain the complete classification of rotational surfaces satisfying (1.2) in the Galilean 3-space.

\section{Preliminaries}

First, we would like to give a brief summary of basic definitions, facts and equations in the theory of surfaces of Galilean 3-space (see for details, [21, 22]).

The Galilean 3 -space $\mathbb{G}^{3}$ arises in a Cayley-Klein way by pointing out an absolute figure $\{\omega, f, J\}$ in the 3 -dimensional real projective space $\mathbb{P}_{3}(\mathbb{R})$. Here $\omega$ is the ideal (absolute) plane, $f$ is the absolute line and $J$ is the fixed elliptic involution of points of $f$. Then the homogeneous coordinates $\left(x_{0}: x_{1}: x_{2}: x_{3}\right) \neq(0: 0: 0: 0)$ are introduced such that $\omega$ is given by $x_{0}=0, f$ is given by $x_{0}=x_{1}=0$ and $J$, by $\left(0: 0: x_{2}: x_{3}\right) \mapsto\left(0: 0:-x_{3}: x_{2}\right)$.

In affine coordinates defined by $\left(x_{0}: x_{1}: x_{2}: x_{3}\right)=\left(1: x_{1}: x_{2}: x_{3}\right)$, the distance between two points $P_{i}=\left(x_{i}, y_{i}, z_{i}\right)$ with $i \in\{1,2\}$ is defined by

$$
d\left(P_{1}, P_{2}\right)=\left\{\begin{array}{ccc}
\left|x_{2}-x_{1}\right| & \text { if } & x_{1} \neq x_{2}, \\
\sqrt{\left(y_{2}-y_{1}\right)^{2}+\left(z_{2}-z_{1}\right)^{2}} & \text { if } & x_{1}=x_{2}
\end{array}\right.
$$


The group of motions of $\mathbb{G}^{3}$ is a six-parameter group. Regarding this group of motions, except the absolute plane, there exist two classes of planes in $\mathbb{G}^{3}$ : Euclidean planes that contain $f$ and in which the induced metric is Euclidean and isotropic planes that do not contain $f$ and in which the induced metric is isotropic. Also, there are four types of lines in $\mathbb{G}^{3}$ : isotropic lines that intersect $f$, non-isotropic lines that do not intersect $f$, non-isotropic lines in $\omega$ and the absolute line $f$ (see [10]).

Let $\vec{X}=\left(x_{1}, x_{2}, x_{3}\right)$ be a vector in $\mathbb{G}^{3}$. If $x_{1}=0$, then $\vec{X}$ is called isotropic, otherwise non-isotropic. Note that, the $x_{1}$-axis is non-isotropic while the $x_{2}$-axis and the $x_{3}$-axis are isotropic, for standard coordinates $\left(x_{1}, x_{2}, x_{3}\right)$. Morever, a plane of the form $x_{1}=$ const. is called an Euclidean plane, otherwise isotropic. For two vectors $\vec{X}=\left(x_{1}, x_{2}, x_{3}\right)$ and $\vec{Y}=\left(y_{1}, y_{2}, y_{3}\right)$, the Galilean scalar product is calculated by

$$
\langle\vec{X}, \vec{Y}\rangle=\left\{\begin{array}{cc}
x_{1} y_{1} & \text { if } \quad x_{1} \neq 0 \quad \text { or } \quad y_{1} \neq 0 \\
x_{2} y_{2}+x_{3} y_{3} & \text { if } \quad x_{1}=y_{1}=0
\end{array}\right.
$$

The norm of vector $\vec{X}$ in $\mathbb{G}^{3}$ is defined by $\|\vec{X}\|:=\sqrt{\langle\vec{X}, \vec{X}\rangle}$. If $\|\vec{X}\|=1$, then $\vec{X}$ is called a unit vector. Also, the Galilean cross product of the vectors $\vec{X}$ and $\vec{Y}$ being at least one is non-isotropic is defined by

$$
\vec{X} \times \vec{Y}=\left(0, x_{3} y_{1}-x_{1} y_{3}, x_{1} y_{2}-x_{2} y_{1}\right) .
$$

Assume that $U$ is an open set of $\mathbb{R}^{2}$ and $S$ is a $C^{r}$-surface such that $r \geq 2$, immersed in $\mathbb{G}^{3}$ parametrized by

$$
\varphi: U \rightarrow \mathbb{R}^{2}, \quad \varphi\left(u_{1}, u_{2}\right)=\left(\varphi_{1}\left(u_{1}, u_{2}\right), \varphi_{2}\left(u_{1}, u_{2}\right), \varphi_{3}\left(u_{1}, u_{2}\right)\right) .
$$

Let us denote $\frac{\partial \varphi}{\partial u_{i}}=\varphi_{, i}, \quad \frac{\partial \varphi_{k}}{\partial u_{i}}=\left(\varphi_{k}\right)_{, i}$ and $\frac{\partial^{2} \varphi_{k}}{\partial u_{i} \partial u_{j}}=\left(\varphi_{k}\right)_{, i j}$ such that $1 \leq k \leq 3$ and $1 \leq i, j \leq 2$. Then a surface is admissible (i.e., without Euclidean tangent planes) if and only if $\left(\varphi_{1}\right)_{u_{i}} \neq 0$ for some $i=1,2$. Let $S \subset \mathbb{G}^{3}$ be a regular admissible surface. We define a side tangential vector by

$$
\sigma=\frac{\left(\varphi_{1}\right)_{, 1} \varphi_{, 2}-\left(\varphi_{1}\right)_{, 2} \varphi_{, 1}}{W}
$$

and a unit normal vector $N$ as

$$
N=\frac{\varphi, 1 \times \varphi, 2}{W}
$$

where the function $W=\left\|\varphi_{, 1} \times \varphi_{, 2}\right\|$ (see [19]).

Now, we introduce the second fundamental coefficients

$$
L_{i j}=\left\langle\frac{\varphi_{, i j}\left(\varphi_{1}\right)_{, 1}-\left(\varphi_{1}\right)_{, i j} \varphi_{, 1}}{\left(\varphi_{1}\right)_{1}}, N\right\rangle=\left\langle\frac{\varphi_{, i j}\left(\varphi_{1}\right)_{, 2}-\left(\varphi_{1}\right)_{, i j} \varphi_{, 2}}{\left(\varphi_{1}\right)_{, 2}}, N\right\rangle
$$

by which the Gaussian curvature $K$ and the mean curvature $H$ of the surface can be written by

$$
K=\frac{L_{11} L_{22}-L_{12}^{2}}{W^{2}}, \quad H=\frac{1}{2} \sum_{i, j=1}^{2} g^{i j} L_{i j}
$$

where

$$
g^{1}=\frac{\left(\varphi_{1}\right)_{2}}{W}, \quad g^{2}=\frac{\left(\varphi_{1}\right)_{1}}{W}, \quad \text { and } \quad g^{i j}=g^{i} g^{j} \quad \text { for } \quad i, j \in\{1,2\}
$$

(see [10]). 


\subsection{Rotation surfaces in $\mathbb{G}^{3}$}

In Galilean 3 -space $\mathbb{G}^{3}$, there are two types of rotations. A Euclidean rotation is given by

$$
\begin{array}{r}
x^{\prime}=x, \\
y^{\prime}=\cos \alpha y+\sin \alpha z, \\
z^{\prime}=-\sin \alpha y+\cos \alpha z .
\end{array}
$$

where $\alpha$ is the Euclidean angle. An isotropic rotation is given by

$$
\begin{array}{r}
x^{\prime}=x+b \alpha, \\
y^{\prime}=y+\alpha x+\frac{b \alpha^{2}}{2}, \\
z^{\prime}=z
\end{array}
$$

where $\alpha$ is called the isotropic angle and $b$ is a positive constant.

Now we would like to give the following definition of a rotational surface in $\mathbb{G}^{3}$ given in [10]:

Definition 2.1. A rotational surface in $\mathbb{G}^{3}$ is a surface that is traced out by a planar curve, the profile curve, rotated in $\mathbb{G}^{3}$. The rotation is either a Euclidean rotation about an axis in the supporting plane of the profile curve, or an isotropic rotation for which a bundle of fixed planes is chosen, [10].

From the definition given above, a rotational surface in $\mathbb{G}^{3}$ is the orbit of points on a profile curve $\gamma$ lying on a plane, under a subgroup $G$ of rotations in $\mathbb{G}^{3}$. Note that a plane is said to be isotropic if it contains both of isotropic and non-isotropic vectors. Otherwise it is called Euclidean. By considering this difference, when $G$ is chosen to be a subset of isotropic rotations, $\gamma$ can be contained in either an Euclidean plane or an isotropic plane. On the other hand, if $G$ is a subset of Euclidean rotations in $\mathbb{G}^{3}$, then $\gamma$ must lie on an Euclidean plane. Therefore, there exists three types of rotational surface in Galilean 3 -space, [10] as follows:

Type I. By isotropic rotating an isotropic profile curve $\gamma(t)=(0, f(t), g(t))$ lying on the Euclidean $y z$-plane, then a type I rotational surface is parametrized by

$$
\varphi(s, t)=\left(b s, f(t)+\frac{b}{2} s^{2}, g(t)\right) .
$$

Type II. By isotropic rotating an non-isotropic profile curve $\gamma(t)=(f(t), g(t), 0)$ lying on the isotropic $x y$-plane, then a type II rotational surface is parametrized by

$$
\varphi(s, t)=\left(f(t)+b s, g(t), s f(t)+\frac{b}{2} s^{2}\right)
$$

where $f$ and $g$ are real functions and $b$ is a positive constant.

Type III. By Euclidean rotating an non-isotropic profile curve $\gamma(t)=(f(t), g(t), 0)$ lying on the isotropic $x y$-plane, one can get a type III rotational surface parametrized by

$$
\varphi(s, t)=(f(t), g(t) \cos s, g(t) \sin s)
$$

where $f$ and $g$ are real functions. 


\section{Classification of rotational surfaces with Cheng-Yau operator in $\mathbb{G}^{3}$}

In this section, we obtain complete classification of rotational surfaces satisfying (1.2) in $\mathbb{G}^{3}$.

\subsection{Rotational surface of Type I}

Let $S$ be an admissible surface in $\mathbb{G}^{3}$ defined by (2.9) with the profile curve $\gamma(t)=$ $(0, f(t), g(t))$. Up to isometries of $\mathbb{G}^{3}$, we may assume $b=1$. Also, the profile curve can be chosen to be

$$
f^{\prime 2}(t)+g^{\prime 2}(t)=1
$$

where $/$ is the ordinary derivative corresponding variable. Moreover, the adapted frame $\left\{e_{1}, e_{2}, G\right\}$ on the rotational surface $S$ is given by

$$
\begin{array}{r}
e_{1}=\varphi_{s}=(1, s, 0), \\
e_{2}=\frac{\varphi_{t}}{\left\|\varphi_{t}\right\|}=\left(0, f^{\prime}(t), g^{\prime}(t)\right), \\
G=e_{1} \times e_{2}=\left(0,-g^{\prime}(t), f^{\prime}(t)\right) .
\end{array}
$$

By (2.6), the Gaussian curvature $K$ and the mean curvature $H$ of $S$ are given by

$$
K=g^{\prime}\left(f^{\prime \prime} g^{\prime}-f^{\prime} g^{\prime \prime}\right) \quad \text { and } \quad H=\frac{f^{\prime} g^{\prime \prime}-f^{\prime \prime} g^{\prime}}{2} .
$$

Now, we put $f^{\prime}(t)=a(t)$ and (3.1) implies $g^{\prime}(t)=\sqrt{1-a^{2}(t)}$. Then (3.3), (3.4) and (3.5) turn into

$$
\begin{array}{r}
e_{2}=\left(0, a(t), \sqrt{1-a^{2}(t)}\right), \\
G=\left(0,-\sqrt{1-a^{2}(t)}, a(t)\right), \\
K=a^{\prime}(t), \\
H=\frac{-a^{\prime}(t)}{2 \sqrt{1-a^{2}(t)}} .
\end{array}
$$

Thus, by using (1.3), we have

$$
L_{1} G=-a^{\prime \prime}(t) e_{2}+\frac{a^{\prime}(t)^{2}}{\sqrt{1-a^{2}(t)}} G .
$$

3.1.1. $L_{1}$-harmonic Gauss map. Let $S$ be a rotational surface defined in (2.9) in $\mathbb{G}^{3}$. Suppose that $S$ has $L_{1}$-harmonic Gauss map, i.e., $L_{1} G=0$. Then from (3.10), we conclude $a^{\prime}(t)=0$. Therefore, (3.8) and (3.9) give $K=H=0$. Hence we have, the following.

Theorem 3.1. An admissible surface $S$ defined in (2.9) in $\mathbb{G}^{3}$ has $L_{1}$-harmonic Gauss map if and only if it is flat and minimal.

Furthermore, by combining Theorem 3.1 with [10, Theorem 4.2], we have directly the following result.

Theorem 3.2. Let $S$ be a rotational surface defined in (2.9) in $\mathbb{G}^{3}$. Then $S$ has $L_{1}$-harmonic Gauss map if and only if it is an open part of a parabolic cylinder or an isotropic plane parametrized by a family of parabolas. 
3.1.2. $L_{1}$-pointwise 1-type Gauss map of the first kind. First, we are going to consider $L_{1}$-pointwise 1-type Gauss map of the first kind. Let $S$ be a rotational surface defined in (2.9) in $\mathbb{G}^{3}$. Then from (3.10), we have

$$
-a^{\prime \prime}(t) e_{2}+\frac{a^{\prime}(t)^{2}}{\sqrt{1-a^{2}(t)}} G=F G,
$$

which implies that $a^{\prime \prime}(t)=0$ and $a^{\prime}(t) \neq 0$. Thus, we get $a^{\prime}(t)=K_{0}$ following that the Gaussian curvature $K$ of $S$ is a non-zero constant. Thus, by considering the assumption $f^{\prime}(t)=a(t)$ and (3.1), the smooth functions $f, g$ are given by

$$
\begin{array}{r}
f(t)=\frac{K_{0} t^{2}}{2}+c t+d, \\
g(t)=\frac{1}{2 K_{0}}\left[\sin ^{-1}\left(K_{0} t+c\right)+\left(K_{0} t+c\right) \sqrt{1-\left(K_{0} t+c\right)^{2}}+d_{0}\right],
\end{array}
$$

where $K_{0}, c, d, d_{0} \in \mathbb{R}_{0}$. So, we have the following theorem:

Theorem 3.3. Let $S$ be a rotational surface defined by $(2.9)$ in $\mathbb{G}^{3}$. Then $S$ has $L_{1}$-pointwise 1-type Gauss map of the first kind if and only if $S$ is parametrized by

$$
\varphi(s, t)=\left(s, f(t)+\frac{s^{2}}{2}, g(t)\right),
$$

where the smooth functions $f, g$ are given in (3.11).

Corollary 3.4. If a rotational surface defined by (2.9) in $\mathbb{G}^{3}$ has $L_{1}$-pointwise 1-type Gauss map of the first kind, then (1.2) is satisfied for $C=0$ and $F=\frac{K_{0}{ }^{2}}{\sqrt{1-\left(K_{0} t+C_{0}\right)^{2}}}$ with $K_{0}, C_{0} \in \mathbb{R}_{0}$.

3.1.3. $L_{1}$-pointwise 1-type Gauss map of the second kind. Now, we state, type I rotational surface with $L_{1}$-pointwise 1-type Gauss map of the second kind. Let $S$ be a rotational surface defined in $(2.9)$ in $\mathbb{G}^{3}$ and suppose that it has $L_{1}$-pointwise 1-type Gauss map of the second kind. Then (1.2) is satisfied for $F \neq 0$ and $C=\left(C_{1}, C_{2}, C_{3}\right) \neq 0$. So, (1.2) and (3.10) give

$$
\begin{array}{r}
-a^{\prime \prime}(t)=F\left\langle C, e_{2}\right\rangle, \\
\frac{a^{\prime 2}(t)}{\sqrt{1-a^{2}(t)}}=F(1+\langle C, G\rangle), \\
\left\langle C, e_{1}\right\rangle=0 .
\end{array}
$$

From (3.2) and (3.12c), we get directly $C_{1}=0$. On the other hand, if we combine (3.12a) and $(3.12 \mathrm{~b})$ with $(3.6)$ and $(3.7)$, we get

$$
\begin{array}{r}
F\left(C_{2} a(t)+C_{3} \sqrt{1-a^{2}(t)}\right)=-a^{\prime \prime}(t), \\
F\left(-C_{2} \sqrt{1-a^{2}(t)}+C_{3} a(t)+1\right)=\frac{a^{\prime}(t)^{2}}{\sqrt{1-a^{2}(t)}},
\end{array}
$$

which yield that the function $a(t)$ satisfies

$$
C_{2}\left(\frac{a(t) a^{\prime 2}(t)-a^{\prime \prime}(t)\left(a(t)^{2}-1\right)}{\sqrt{1-a^{2}(t)}}\right)+C_{3}\left(a^{\prime 2}(t)+a^{\prime \prime}(t) a(t)\right)+a^{\prime \prime}(t)=0 .
$$

Therefore, we have the following theorem: 
Theorem 3.5. Let $S$ be a rotational surface defined by $(2.9)$ in $\mathbb{G}^{3}$. Then $S$ has $L_{1}$-pointwise 1-type Gauss map of the second kind if and only if $S$ is parametrized by

$$
\varphi(s, t)=\left(s, \int^{t} a(\tau) d \tau+\frac{1}{2} s^{2}, \int^{t} \sqrt{1-a^{2}(\tau)} d \tau\right)
$$

where the smooth function a(t) satisfies (3.13).

Corollary 3.6. Let $S$ be a rotational surface defined by (2.9) in $\mathbb{G}^{3}$. If (1.2) is satisfied for $C=\left(0,0, C_{3}\right) \neq 0$, then the smooth functions $f, g$ are given by

$$
\begin{array}{r}
f(t)=\frac{1}{C_{3}}\left(-t+\frac{1}{3 C_{3} d_{1}}\left(1+2 C_{3}\left(d_{1} t+d_{2}\right)\right)^{3 / 2}\right)+d_{3}, \\
g(t)= \pm \int\left(1+\left(-\frac{1}{C_{3}} \pm \frac{1}{C_{3}} \sqrt{1+2 C_{3}\left(d_{1} t+d_{2}\right)}\right)^{2}\right)^{1 / 2} d t .
\end{array}
$$

Proof. Assume that $C=\left(0,0, C_{3}\right)$. By considering this assumption in (3.13), we obtain the following ODE,

$$
C_{3}\left(a^{\prime}(t)\right)^{2}+a^{\prime \prime}(t)\left(1+C_{3} a(t)\right)=0
$$

whose general solution is given by

$$
a(t)=-\frac{1}{C_{3}} \pm \frac{1}{C_{3}} \sqrt{1+2 C_{3}\left(d_{1} t+d_{2}\right)},
$$

where $d_{1}, d_{2} \in \mathbb{R}$. By considering the assumption $f^{\prime}(t)=a(t)$ and (3.1), the smooth functions $f, g$ are obtained as in (3.14).

\subsection{Rotational surface of Type II}

Let $S$ be an admissible surface in $\mathbb{G}^{3}$ defined in (2.10). Without loss of generality, we may assume that $b=1$ and that the profile curve $\gamma(t)=(f(t), g(t), 0)$ is parametrized by arc-length. So the surface $S$ can be rewritten as

$$
\varphi(s, t)=\left(t+s, g(t), s t+\frac{s^{2}}{2}\right) .
$$

The adapted frame $\left\{\sigma, e_{2}, G\right\}$ on the rotational surface $S$ is given by

$$
\begin{gathered}
\sigma=\frac{\left(0, g^{\prime}(t),-t\right)}{\sqrt{t^{2}+g^{\prime}(t)^{2}}}, \\
e_{2}=\left(1, g^{\prime}(t), s\right), \\
G=\frac{\left(0, t, g^{\prime}(t)\right)}{\sqrt{t^{2}+g^{\prime}(t)^{2}}} .
\end{gathered}
$$

By (2.6), the Gaussian curvature $K$ and the mean curvature $H$ of $S$ are given by

$$
\begin{array}{r}
K=\frac{g^{\prime}(t)\left(t g^{\prime \prime}(t)-g^{\prime}(t)\right)}{\left(t^{2}+g^{\prime}(t)^{2}\right)^{2}}, \\
H=\frac{t g^{\prime \prime}(t)-g^{\prime}(t)}{2{\sqrt{t^{2}+g^{\prime}(t)^{2}}}^{3 / 2}} .
\end{array}
$$

Therefore, the operator $L_{1}$ of the Gauss map given in (1.3) can be expressed as

$$
L_{1} G=-K^{\prime}(t) e_{2}+\frac{g^{\prime}(t)\left(t g^{\prime \prime}(t)-g^{\prime}(t)\right)^{2}}{\left(t^{2}+g^{\prime}(t)^{2}\right)^{7 / 2}} G .
$$


3.2.1. $L_{1}$-harmonic Gauss map. Let $S$ be a rotational surface of type II defined in (2.10). Suppose that $L_{1} G=0$. Then from (3.22), we get

$$
K^{\prime}(t)=0 \quad \text { and } \quad g^{\prime}(t)\left(t g^{\prime \prime}(t)-g^{\prime}(t)\right)=0 .
$$

Therefore, (3.21a) and (3.21b) give $K=H=0$. Hence, we have the following result.

Theorem 3.7. An admissible surface $S$ defined in (2.10) in $\mathbb{G}^{3}$ has $L_{1}$-harmonic Gauss map if and only if it is flat and minimal.

Moreover, by combining Theorem 3.7 with [10, Theorem 4.6], we have

Theorem 3.8. Let $S$ be a rotational surface of type II defined in (2.10) in $\mathbb{G}^{3}$. Then $S$ has $L_{1}$-harmonic Gauss map if and only if it is an open part of an isotropic plane parametrized by a family of parabolas, a parabolic cylinder or a cyclic surface (parabolic sphere).

3.2.2. $L_{1}$-pointwise 1-type Gauss map of the first kind. Let $S$ be a rotational surface of type II, defined in (2.10), with $L_{1}$-pointwise 1-type Gauss map of the first kind. Then from (3.22), we have

$$
K^{\prime}(t)=0 \quad \text { and } \quad-\frac{g^{\prime}(t)\left(t g^{\prime \prime}(t)-g^{\prime}(t)\right)^{2}}{\left(t^{2}+g^{\prime}(t)^{2}\right)^{7 / 2}}=F .
$$

From the first equation above, we get $K(t)=K_{0}$ being non-zero constant. By considering this result in the first equation given in (3.21a), we obtain the following ODE

$$
g^{\prime}(t)\left(t g^{\prime \prime}(t)-g^{\prime}(t)\right)=K_{0}\left(t^{2}+g^{\prime}(t)^{2}\right)^{2} .
$$

To solve (3.23), we set $g^{\prime}(t)=y(t)$; then

$$
y\left(t y^{\prime}-y\right)=K_{0}\left(t^{2}+y^{2}\right)^{2}
$$

whose general solution is

$$
y(t)= \pm \frac{\sqrt{-K_{0}\left(t^{2}+2 c\right)\left(1+K_{0}\left(t^{2}+2 c\right)\right)} t}{K_{0}\left(t^{2}+2 c\right)},
$$

where $K_{0}, c \in \mathbb{R}_{0}$. Hence, the obtained solution was considered into the assumption $g^{\prime}(t)=y(t)$, we get

$$
g(t)= \pm 2 c \cosh ^{-1}\left(u(t) \mp \frac{\sqrt{u^{2}(t)-1}}{2 K_{0}}\right),
$$

where $u(t)=K_{0} t^{2}-\frac{1}{2}$. So, we have the following theorem:

Theorem 3.9. Let $S$ be a rotational surface defined by $(2.10)$ in $\mathbb{G}^{3}$. Then $S$ has $L_{1}$-pointwise 1-type Gauss map of the first kind if and only if $S$ is parametrized by

$$
\varphi(s, t)=\left(t+s, g(t), s t+\frac{s^{2}}{2}\right),
$$

where the smooth functions $g$ are given in (3.24).

3.2.3. $L_{1}$-pointwise 1-type Gauss map of the second kind. Let $S$ be a rotational surface of type II defined in (2.10). Assume that it has $L_{1}$-pointwise 1-type Gauss map of the second kind. Then (1.2) is satisfied for $C=\left(C_{1}, C_{2}, C_{3}\right) \neq 0$ and $F \neq 0$. From (1.2) and (3.22), we have

$$
-K^{\prime}(t) e_{2}+\frac{g^{\prime}(t)\left(t g^{\prime \prime}(t)-g^{\prime}(t)\right)^{2}}{\left(t^{2}+g^{\prime}(t)^{2}\right)^{7 / 2}} G=F(G+C),
$$


which implies

$$
\begin{array}{r}
-K^{\prime}(t)=F\left\langle C, e_{2}\right\rangle, \\
-\frac{g^{\prime}\left(t g^{\prime \prime}-g^{\prime}\right)^{2}}{\left(t^{2}+g^{\prime 2}(t)\right)^{7 / 2}}=F(1+\langle C, G\rangle), \\
0=\langle C, \sigma\rangle .
\end{array}
$$

Let us distinguish the following cases:

(1) Assume $C_{1}=0$. Then (3.19) and (3.26a) implies $K^{\prime}=0$. On the other hand, (3.20) and (3.26b) give $F=\frac{g^{\prime}(t)\left(t g^{\prime \prime}(t)-g^{\prime}(t)\right)^{2}}{\left(t^{2}+g^{\prime}(t)^{2}\right)^{7 / 2}}$. Therefore (3.25) implies $C=0$, which is a contradiction.

(2) If $C_{1} \neq 0$, then from (3.19) and (3.26a), we rewrite the equation (3.26a) as

$$
-\frac{K^{\prime}(t)}{C_{1}}=F
$$

On the other hand, from (3.20) and (3.26b) we obtain,

$$
-\frac{g^{\prime}\left(t g^{\prime \prime}-g^{\prime}\right)^{2}}{\left(t^{2}+g^{\prime 2}(t)\right)^{7 / 2}}=F \text {. }
$$

Therefore (3.25), (3.27) and (3.28) imply $F\left(G+e_{2}\right)=F(G+C)$, which gives $C=e_{2}$, which is imposible. Hence, $S$ can not have $L_{1}$-pointwise 1-type Gauss map of the second kind.

Therefore, we have the following theorem:

Theorem 3.10. There is no a type II rotational surface defined by (2.10), generated by a non-isotropic curve in $\mathbb{G}^{3}$ with $L_{1}$-pointwise 1-type Gauss map of the second kind.

\subsection{Rotational surface of Type III}

Let $S$ be an admissible surface in $\mathbb{G}^{3}$ defined in (2.11). Up to isometries of $\mathbb{G}^{3}$, we may assume that the non-isotropic profile curve $\gamma(t)=(f(t), g(t), 0)$ is parametrized by arc-length, i.e., $f(t)=t$. Thus the surface $S$ can be reparametrized as

$$
\varphi(s, t)=(t, g(t) \cos s,-g(t) \sin s) .
$$

Thus, the orthonormal frame $\left\{e_{1}, e_{2}\right\}$ on the tangent plane of $S$ is given by

$$
\begin{array}{r}
e_{1}=\frac{\varphi_{s}}{\left\|\varphi_{s}\right\|}=(0,-\sin s,-\cos s), \\
e_{2}=\frac{\varphi_{t}}{\left\|\varphi_{t}\right\|}=\left(1, g^{\prime}(t) \cos s,-g^{\prime}(t) \sin s\right) .
\end{array}
$$

Moreover, the Gauss map of $S$ is

$$
G=e_{1} \times e_{2}=(0,-\cos s, \sin s) .
$$

Also by(2.6), the Gaussian curvature $K$ and the mean curvature $H$ of $S$ are given by

$$
K=\frac{-g^{\prime \prime}(t)}{g(t)} \quad \text { and } \quad H=-\frac{1}{2 g(t)} .
$$

Thus, the Cheng-Yau operator $L_{1}$ of the Gauss map given in (1.3) can be expressed as

$$
L_{1} G=\left[\frac{g^{\prime \prime}(t)}{g(t)}\right]^{\prime} e_{2}+\frac{g^{\prime \prime}(t)}{2 g(t)^{2}} G .
$$


3.3.1. $L_{1}$-harmonic Gauss map. Let $S$ be a rotational surface defined in $(2.11)$ in $\mathbb{G}^{3}$. Assume that $L_{1} G=0$. Then from (3.34), we get directly $g(t)=c_{0} t+c_{1}$ with $c_{0}, c_{1} \in \mathbb{R}$. By considering this result the first expression in (3.33), we get $K=0$. Therefore, we have the following.

Theorem 3.11. An admissible surface $S$ defined in (2.11) in $\mathbb{G}^{3}$ has $L_{1}$-harmonic Gauss map if and only if it is flat.

Also, by combining Theorem 3.11 with [10, Theorem 4.9], we have the following result.

Theorem 3.12. Let $S$ be a type III rotational surface defined in (2.11) in $\mathbb{G}^{3}$. Then $S$ has $L_{1}$-harmonic Gauss map if and only if it is an open part of a cylinder over an Euclidean circle or a circular coni.

3.3.2. $L_{1}$-pointwise 1-type Gauss map of the first kind. Let $S$ be a rotational surface of type III defined in (2.11) in $\mathbb{G}^{3}$. Suppose that $S$ has $L_{1}$-pointwise 1-type Gauss map of the first kind. Then from (3.34) and $L_{1} G=F G$, we have

$$
g^{\prime \prime \prime}(t) g(t)-g^{\prime \prime}(t) g^{\prime}(t)=0 \quad \text { and } \quad \frac{g^{\prime \prime}(t)}{g(t)^{2}}=F .
$$

From the first equation given above, we conclude

$$
g^{\prime \prime}(t)=K_{0} g(t), \quad K_{0} \in \mathbb{R}
$$

Therefore, from the first equation given in (3.33), we have the surface $S$ has non-zero constant Gaussian curvature.

Now, if $K_{0}$ is positive, then the general solution of (3.35) is

$$
g(t)=A \sinh \left(\sqrt{K_{0}} t\right)+B \cosh \left(\sqrt{K_{0}} t\right),
$$

otherwise,

$$
g(t)=A \sin \left(\sqrt{K_{0}} t\right)+B \cos \left(\sqrt{K_{0}} t\right)
$$

where $A, B \in \mathbb{R}$. Thus, we have the following theorem:

Theorem 3.13. Let $S$ be a rotational surface of type III defined in (2.11) in $\mathbb{G}^{3}$. Then $S$ has $L_{1}$-pointwise 1-type Gauss map of the first kind if and only if $S$ is parametrized by

$$
\varphi(s, t)=(t, g(t) \cos s,-g(t) \sin s),
$$

where the function $g(t)$ is given in (3.36) or (3.37).

3.3.3. $L_{1}$-pointwise 1-type Gauss map of the second kind. Assume that $S$ is a rotational surface of type III defined in $(2.11)$ in $\mathbb{G}^{3}$ and that it has $L_{1}$-pointwise 1-type Gauss map of the second kind. By considering the conditions (1.2) and (3.34) together, we get

$$
\begin{array}{r}
\frac{g^{\prime \prime}(t) g^{\prime}(t)-g^{\prime \prime \prime}(t) g^{\prime}(t)}{g^{2}(t)}= \\
\frac{g^{\prime \prime}(t)}{g^{2}(t)}=F\left(1+e_{2}\right\rangle, \\
0=\left\langle C, e_{1}\right\rangle,
\end{array}
$$

where $F$ is a non-zero smooth function and $C=\left(C_{1}, C_{2}, C_{3}\right)$ is a constant vector in $\mathbb{G}^{3}$. Let us distinguish the following cases:

(1) If $C_{1}=0$, then from $(3.38 \mathrm{c})$ and $(3.30)$, we get

$$
C_{2} \sin s+C_{3} \cos s=0 .
$$

As $\{\sin s, \cos s\}$ forms a set of linearly dependent functions, we get

$$
C_{2}=0 \quad \text { and } \quad C_{3}=0 .
$$

But this is a contradiction. 
(2) If $C_{1} \neq 0$, then from $\left\langle C, e_{2}\right\rangle=C_{1},\left\langle C, e_{1}\right\rangle=0$ and $\langle C, G\rangle=0$. Thus the constant vector $C$ becomes $C=C_{1} e_{2}$ which implies $g^{\prime}(t)=0$ because of (3.31). However, in this case (3.34) gives $L_{1} G=0$, which is a contradiction.

So, we have the following theorem:

Theorem 3.14. There is no a rotational surface of type III defined in (2.11), generated by a non-isotropic curve in $\mathbb{G}^{3}$ with $L_{1}$-pointwise 1-type Gauss map of the second kind.

\section{References}

[1] L.J. Alias and N. Gurbuz, An extension of Takashi theorem for the linearized operators of the highest order mean curvatures, Geom. Dedicata 121, 113-127, 2006.

[2] M.E. Aydin, A.O. Oğrenmis and M. Ergut, Classification of factorable surfaces in the pseudo-Galilean space, Glas. Mat. Ser. III 50 (2), 441-451, 2015.

[3] B.Y. Chen, Total mean curvature and submanifolds finite type, World Scientific Publ., New Jersey, 1984.

[4] B.Y. Chen, A report on submanifolds of finite type, Soochow J. Math. 22, 117-337, 1996.

[5] B.Y. Chen, M. Choi, and Y.H. Kim, Surfaces of revolution with pointwise 1-type Gauss map, J. Korean Math. Soc. 42, 447-455, 2005.

[6] B.Y. Chen, J. Morvan and T. Nore, Energy, tension and finite type maps, Kodai Math. J. 9, 406-418, 1986.

[7] B.Y. Chen, and P. Piccinni, Submanifolds with finite type Gauss map, Bull. Austral. Math. Soc. 35 (2), 161-186, 1987.

[8] S.Y. Cheng and S.T. Yau, Hypersurfaces with constant scalar curvature, Math. Ann. 225, 195-204, 1977.

[9] M. Dede, Tubular surfaces in Galilean space, Math. Commun. 18, 209-217, 2013.

[10] M. Dede, C. Ekici and W. Goemans, Surfaces of revolution with vanishing curvature in Galilean 3-space, J. Math. Physics, Analysis, Geometry 14 (2), 141-152, 2018.

[11] U. Dursun, Hypersurfaces with pointwise 1-type Gauss map, Taiwanese J. Math. 11 (5), 1407-1416, 2007.

[12] S.M.B. Kashani, On some $L_{1}$ - finite type (hyper)surfaces in $\mathbb{R}^{n+1}$, Bull. Korean Math. Soc. 46 (1), 35-43, 2009.

[13] U.H. Ki, D.S. Kim, Y. H. Kim and Y.M. Roh, Surfaces of revolution with pointwise 1-type Gauss map in Minkowski 3-space, Taiwan. J. Math. 13 (1), 317-338, 2009.

[14] D.S. Kim, J.R. Kim and Y. H. Kim, Cheng-Yau operator and Gauss map of surfaces of revolution, Bull. Malays. Math. Sci. Soc. 39 (4), 1319-1327, 2016.

[15] Y.H. Kim and N.C. Turgay, Surfaces in $\mathbb{E}^{3}$ with $L_{1}$-pointwise 1-type Gauss map, Bull. Korean Math. Soc. 50 (3), 935-949, 2013.

[16] Y.H. Kim and N.C. Turgay, On the helicoidal surfaces in $\mathbb{E}^{3}$ with $L_{1}$-pointwise 1-type Gauss map, Bull. Korean Math. Soc. 50, 1345-1356, 2013.

[17] Y.H. Kim and D.W. Yoon, Ruled surfaces with pointwise 1-type Gauss map, J. Geom. Phys. 34 (3-4), 191-205, 2000.

[18] Z.M. Sipus, Ruled Weingarten surfaces in Galilean space, Period. Math. Hungar. 56, 213-225, 2008.

[19] Z.M. Sipus and B. Divjak, Translation surfaces in the Galilean space, Glas. Mat. Ser. III 46, No. 66, 455-469, 2011.

[20] Z.M. Sipus, and B. Divjak, Surfaces of constant curvature in pseudo-Galilean space, Int. J. Math. Sci. 2012, Art. ID 375264, 28 pp, 2012.

[21] D. Palman, Drehyzykliden des Galileischen Raumes $G_{3}$, Math. Pannon. 2 (1), 98-104, 1991. 
[22] O. Roschel, Die Geometrie des Galileischen Raumes, Bericht der MathematischStatistischen Sektion in der Forschungsgesellschaft Joanneum, Bericht Nr. 256, Habilitationsschrift, Leoben, 1984.

[23] D.W. Yoon, Surfaces of revolution in the three dimensional pseudo-Galilean space, Glas. Mat. Ser. III 48, No. 68, 415-428, 2013.

[24] D.W. Yoon, Y.H. Kim and J.S. Jung, Rotation surfaces with $L_{1}$-pointwise 1-type Gauss map in pseudo-Galilean space, Ann. Polon. Math. 113 (3), 255-267, 2015. 